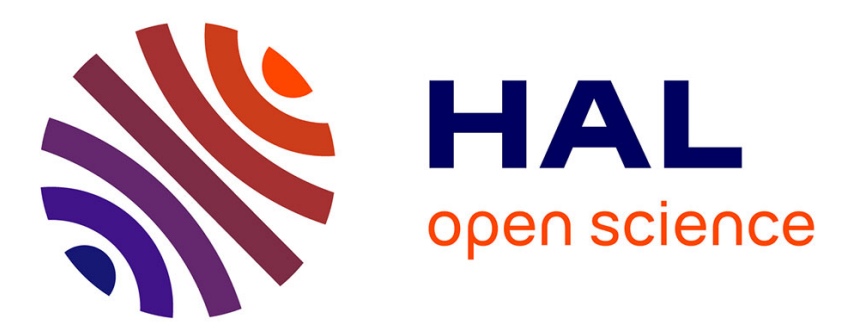

\title{
A homogeneity property of a class of discrete-time systems
}

Tonametl Sanchez, Denis Efimov, Andrey Polyakov, Jaime Moreno, Wilfrid

Perruquetti

\section{> To cite this version:}

Tonametl Sanchez, Denis Efimov, Andrey Polyakov, Jaime Moreno, Wilfrid Perruquetti. A homogeneity property of a class of discrete-time systems. 56th IEEE Conference on Decision and Control (CDC), Dec 2017, Melbourne, Australia. hal-01611972

\section{HAL Id: hal-01611972 \\ https://hal.inria.fr/hal-01611972}

Submitted on 6 Oct 2017

HAL is a multi-disciplinary open access archive for the deposit and dissemination of scientific research documents, whether they are published or not. The documents may come from teaching and research institutions in France or abroad, or from public or private research centers.
L'archive ouverte pluridisciplinaire HAL, est destinée au dépôt et à la diffusion de documents scientifiques de niveau recherche, publiés ou non, émanant des établissements d'enseignement et de recherche français ou étrangers, des laboratoires publics ou privés. 


\title{
A homogeneity property of a class of discrete-time systems
}

\author{
Tonametl Sanchez, Denis Efimov, Andrey Polyakov, Jaime A. Moreno and Wilfrid Perruquetti
}

\begin{abstract}
In this paper we study a dynamic property of a class of discrete-time systems. Such a property, that we call discrete-homogeneity, is verifiable algebraically in the transition map of the system. Discrete-homogeneity allows to establish stability features of the system by considering only the discretehomogeneity degree. Such stability properties are studied by means of Lyapunov and Lyapunov-like functions.
\end{abstract}

\section{INTRODUCTION}

Homogeneity has received significant attention in (continuous-time) Control Systems Theory [15], [6], [8], [12], [13], [5], [11], [10], [2], [4], [3], which is originated by advantages imported by homogeneity in the analysis and design of control systems. The main feature in a homogeneous system is that its trajectories exhibit a dilating property, i.e. a scaling of the initial condition is equivalent to a scaling of the original trajectory. An important consequence of such a feature is that local properties of homogeneous systems can be translated into global ones. Some additional advantages of homogeneity in continuous-time systems are the following: homogeneity can be easily checked as an algebraic property of the vector field of the system; when the system's origin is asymptotically stable, the convergence rate is specified by the homogeneity degree; there exist converse Lyapunov theorems that assert the existence of smooth homogeneous Lyapunov functions for homogeneous systems; the homogeneity degree allows to determine several robustness properties of the system.

On the other hand, discrete-time dynamic systems play an important role in Control Systems Theory. They appear as mathematical models of real life situations [1] or as discretizations of continuous-time systems. The usefulness of homogeneity for continuous-time systems is naturally desired to be extensible to discrete-time systems. Some variants of this extension have been proposed in [7] and [14]. In those papers a concept of homogeneity for discrete-time systems is defined, that concept coincides with the conventional setting in the case of homogeneity of degree zero. It seems that it is the only case when the standard definition of homogeneity (introduced for continuous-time systems) can be applied in the discrete-time framework, with translation of useful properties form continuous-time to discrete-time cases. Therefore, for the discrete-time systems some new concepts have to be introduced.

T. Sanchez and J. A. Moreno are with Instituto de Ingeneiría, Universidad Nacional Autónoma de México, Mexico City, Mexico, TSanchezRQiingen. unam.mx, JMorenoP@ii.unam.mx

D. Efimov, A. Polyakov and W. Perruquetti are with Non-A team, Inria Lille - Nord Europe, France, Denis.Efimoveinria.fr, Andrey.Polyakov@inria.fr, Wilfrid.Perruquetti dinria.fr
In this paper we introduce a definition of homogeneity for discrete-time systems, we call it discrete-homogeneity (D-homogeneity). This definition consists in a small variation of the usual one for vector fields, however, it guarantees some stability properties of the solutions of Dhomogeneous discrete-time systems, granting them many interesting features. In particular, we analyse the stability properties of discrete-time systems whose transition map is D-homogeneous. We show that D-homogeneity directly implies (local) asymptotic stability of the system's origin or global boundedness of the trajectories depending on the Dhomogeneity degree. We also show that when the origin is locally asymptotically stable the growth of the trajectories, starting far enough from the origin, is unbounded. In the case of global boundedness the origin is unstable. Every case can be proven using homogeneous Lyapunov or Lyapunov-like functions. Although the proofs of the main theorems are not provided in this paper, we give some examples showing the usefulness of D-homogeneity, e.g. possible applications of D-homogeneity in the design of controllers for discrete-time systems.

Paper organization: In Section II, Lyapunov theorems for discrete-time systems are recalled. In Section III the definition of D-homogeneity proposed in this paper is stated. The stability properties of D-homogeneous systems are described in Section IV. Some applications of D-homogeneity to the stabilization problem is given in Section V. Finally, some conclusions and possible future research are stated in Section VI.

Notation: Real and Integer numbers are denoted as $\mathbb{R}$ and $\mathbb{Z}$, respectively. $\mathbb{R}_{>0}$ denotes the set $\{x \in \mathbb{R}: x>0\}$, analogously for the set $\mathbb{Z}$ and the sign $\geq$. For $x \in \mathbb{R}^{n},|x|$ denotes the Euclidean norm. $f \circ g$ denotes the composition of two functions (with adequate domains and codomains), i.e., $(f \circ g)(x)=f(g(x))$, thus, we denote $(\underbrace{f \circ f \circ \cdots \circ f}_{r-\text { times }})(x)=$ $f^{[r]}(x)$, for example, $f^{[1]}(x)=f(x)$ and $f^{[2]}(x)=f(f(x))$. For a function $V: \mathbb{R}^{n} \rightarrow \mathbb{R}$ and some $\alpha, \bar{\alpha} \in \mathbb{R}_{>0}, \mathcal{I}(V, \alpha)$ and $\mathcal{E}(V, \bar{\alpha})$ denote the sets $\left\{x \in \mathbb{R}^{n}: V(x) \leq \alpha\right\}$ and $\left\{x \in \mathbb{R}^{n}: V(x) \geq \bar{\alpha}\right\}$, respectively. For $x \in \mathbb{R},\lceil x\rfloor^{q}$ denotes $\operatorname{sign}(x)|x|^{q}$.

\section{LYAPUNOV STABILITY}

In this paper we consider the autonomous discrete-time dynamic system

$$
x(k+1)=f(x(k)),
$$

where the state $x(k) \in \mathbb{R}^{n}$, for any $k \in \mathbb{Z}_{\geq 0}$. We assume that the transition map $f: \mathbb{R}^{n} \rightarrow \mathbb{R}^{n}$ is a continuous function of 
its argument.

Let $F\left(k ; x_{0}\right)$ denote the solution of (1) with initial condition $x_{0}=x(0)$, thus, $F\left(0 ; x_{0}\right)=x_{0}$. Note that

$$
F\left(1 ; x_{0}\right)=f\left(x_{0}\right), \quad F\left(2 ; x_{0}\right)=f\left(F\left(1 ; x_{0}\right)\right), \quad \ldots
$$

therefore, $F\left(k ; x_{0}\right)=f^{[k]}\left(x_{0}\right)$. In this notation, $F\left(0 ; x_{0}\right)=$ $f^{[0]}\left(x_{0}\right)=x_{0}$.

The stability definitions in the sense of Lyapunov for discrete-time systems coincide, in general, with those for continuous-time systems, see e.g. [1, Chapter 5]. We evoke below the characterization of stability through Lyapunov functions. Recall that a equilibrium point of (1) is any point in the space of the states such that it is a solution of the equation $f(x)-x=0$.

Theorem 1 ([6]): Let $x=0$ be an equilibrium point of (1) and let $\mathcal{S} \subset \mathbb{R}^{n}$ be a neighbourhood of $x=0$. If there exist a continuous positive definite function $V: \mathcal{S} \rightarrow \mathbb{R}$ such that

- $V(f(x))-V(x) \leq 0$ for all $x \in \mathcal{S}$, then $x=0$ is a stable equilibrium point of (1),

- $V(f(x))-V(x) \leq-\eta(|x|)$, where $\eta$ is a class $\mathcal{K}$ function, for all $x \in \mathcal{S}$, then $x=0$ is an asymptotically stable equilibrium point of (1).

For this and related theorems see also [1] and the references therein.

\section{Discrete Homogeneity}

As we have said, in this paper we describe a homogeneity property of a class of discrete-time systems, but before we give the definition of D-homogeneity, let us recall the usual definition of weighted homogeneity.

Definition 1 ([8]): Let $\Lambda_{\epsilon}^{\mathbf{r}}$ be the square diagonal matrix given by $\Lambda_{\epsilon}^{\mathbf{r}}=\operatorname{diag}\left(\epsilon^{\mathrm{r}_{1}}, \ldots, \epsilon^{\mathrm{r}_{\mathrm{n}}}\right)$, where $\mathbf{r}=\left[r_{1}, \ldots, r_{n}\right]^{\top}$, $r_{i} \in \mathbb{R}_{>0}$, and $\epsilon \in \mathbb{R}_{>0}$. The components of $\mathbf{r}$ are called the weights of the coordinates. Thus:

a) A function $f: \mathbb{R}^{n} \rightarrow \mathbb{R}$ is $\mathbf{r}$-homogeneous of degree $m \in \mathbb{R}$ if $f\left(\Lambda_{\epsilon}^{\mathbf{r}} x\right)=\epsilon^{m} f(x), \forall x \in \mathbb{R}^{n}, \forall \epsilon \in \mathbb{R}_{>0}$.

b) A vector field $f: \mathbb{R}^{n} \rightarrow \mathbb{R}^{n}, f=\left[f_{1}(x), \ldots, f_{n}(x)\right]^{\top}$, is $\mathbf{r}$-homogeneous of degree $\kappa \in \mathbb{R}$ if $f_{i}\left(\Lambda_{\epsilon}^{\mathbf{r}} x\right)=$ $\epsilon^{\kappa+r_{i}} f_{i}(x), i=1,2, \ldots, n, \forall x \in \mathbb{R}^{n}, \forall \epsilon \in \mathbb{R}_{>0}$.

This definition is used in [7] for discrete-time systems. The definition given in [14] coincides with Definition 1 when dilations $\Lambda_{\epsilon}^{\mathbf{r}}$ are considered. D-homogeneity consists in a modification of the usual definition for vector fields, we state it as follows.

Definition 2 (Discrete-homogeneity): Let $\Lambda_{\epsilon}^{\mathbf{r}}, \mathbf{r}$ and $\epsilon$ be as in Definition 1.

b) A vector field $f: \mathbb{R}^{n} \rightarrow \mathbb{R}^{n}$, with $f(x)=$ $\left[f_{1}(x), \ldots, f_{n}(x)\right]^{\top}$, is $D_{\mathbf{r}}$-homogeneous of degree $\nu \in$ $\mathbb{R}$ if $f_{i}\left(\Lambda_{\epsilon}^{\mathbf{r}} x\right)=\epsilon^{r_{i} \nu} f_{i}(x), i=1,2, \ldots, n, \forall x \in \mathbb{R}^{n}$, $\forall \epsilon \in \mathbb{R}_{>0}$ or equivalently $f\left(\Lambda_{\epsilon}^{\mathbf{r}} x\right)=\left(\Lambda_{\epsilon}^{\mathbf{r}}\right)^{\nu} f(x)=$ $\Lambda_{\epsilon}^{\nu \mathbf{r}} f(x)=\Lambda_{\epsilon^{\nu}}^{\mathbf{r}} f(x)$. c) A system as (1) is $D_{\mathbf{r}}$-homogeneous of degree $\nu \in \mathbb{R}$ if its transition map $f$ is $D_{\mathbf{r}}$-homogeneous of degree $\nu \in \mathbb{R}$.

Observe that we are not modifying the definition for scalar functions. Also note that, for vector fields, both definitions are equivalent when $\kappa=0$ and $\nu=1$. Thus, definition of D-homogeneity of degree one for (1) coincides with the homogeneity of degree zero given in [7] and [14]. Let us recall the following result on the solutions of homogeneous (continuous-time) systems.

Lemma 1 (see e.g. [15]): Consider the system $\dot{x}=f(x)$, $x \in \mathbb{R}^{n}$, and suppose that $f$ is $\mathbf{r}$-homogeneous of degree $\kappa$. Let $\phi\left(t ; x_{0}\right)$ denote a system's solution with initial condition $x_{0}=x(0)$, then

$$
\phi\left(t ; \Lambda_{\epsilon}^{\mathbf{r}} x_{0}\right)=\Lambda_{\epsilon}^{\mathbf{r}} \phi\left(\epsilon^{\kappa} t ; x_{0}\right)
$$

From this lemma, the scaling property of the solutions of a homogeneous systems is evident. The extension of this result for homogeneous differential inclusions can be consulted in [10], [3]. Now we give an analogous result for D-homogeneous discrete-time systems.

Lemma 2: If (1) is $D_{\mathbf{r}}$-homogeneous of degree $\nu$, then its solutions $F\left(k ; x_{0}\right)$ are such that

$$
F\left(k ; \Lambda_{\epsilon}^{\mathbf{r}} x_{0}\right)=\left\{\begin{array}{cl}
\Lambda_{\epsilon}^{\mathbf{r}} x_{0}, & k=0, \\
\left(\Lambda_{\epsilon}^{\mathbf{r}}\right)^{k \nu} F\left(k ; x_{0}\right), & k \in \mathbb{Z}_{>0} .
\end{array}\right.
$$

Remark 1: As in the continuous-time case, Dhomogeneous discrete-time systems admit scaling of their trajectories.

\section{Stability PROPERTIES OF D-HOMOGENEOUS SYSTEMS}

In this section, (1) is assumed to be D-homogeneous of some degree $\nu$ with a unique equilibrium point at the origin. We show that the value of $\nu$ implies directly stability features of (1) for the cases $\nu>1$ and $\nu \in(0,1)$.

\section{A. D-homogeneous systems of degree $\nu>1$}

Theorem 2: Suppose that (1) is $D_{\mathbf{r}}$-homogeneous of degree $\nu>1$, with a unique equilibrium point at $x=0$. Let $V$ : $\mathbb{R}^{n} \rightarrow \mathbb{R}$ be any continuous positive definite $\mathbf{r}$-homogeneous function of any degree $m \in \mathbb{R}_{>0}$. The following hold.

- $x=0$ is an (locally) asymptotically stable equilibrium point of (1), and there exists $\alpha \in \mathbb{R}_{>0}$ such that $V$ is a Lyapunov function for (1) on $\mathcal{I}(V, \alpha)$.

- If $f(x)=0 \Leftrightarrow x=0$, there exists $\bar{\alpha} \in \mathbb{R}_{>0}$ such that the trajectories $F\left(k ; x_{0}\right)$ of (1) are unbounded for any $x_{0} \in \mathcal{E}(V, \bar{\alpha})$.

From Theorem 2 we can make the following observations:

- To ensure (local) asymptotic stability of the origin of (1), it is sufficient to verify D-homogeneity of degree $\nu>1$. 
- Theorem 2 guarantees that any $\mathbf{r}$-homogeneous positive definite function $V$ of degree $m \in \mathbb{R}_{>0}$ (with weights $\mathbf{r}$ ) is a Lyapunov function for (1).

- D-homogeneity degree $\nu>1$ guarantees asymptotic stability of $x=0$ but prevents it to be a globally stable equilibrium point of (1) under the condition $f(x)=0 \Leftrightarrow x=0$, moreover, in this case, the trajectories starting far enough from the origin cannot remain bounded.

\section{B. D-homogeneous systems of degree $\nu \in(0,1)$}

For the definition of practical stability used in the following theorem see e.g. [1, Chapter 5]

Theorem 3: If (1) is $D_{\mathbf{r}}$-homogeneous of degree $\nu \in$ $(0,1)$ with a unique equilibrium point at $x=0$, then the following hold.

- The origin of (1) is practically stable and for any bounded initial condition, the trajectories of (1) enter the practical-stability region $\mathcal{S}$ in a finite number of steps.

- If in addition $f(x)=0 \Leftrightarrow x=0$, the origin of (1) is locally unstable.

From Theorem 3 we can observe the following:

- Condition $\nu \in(0,1)$ guarantees that for any bounded initial condition the trajectories of the system enter a vicinity of the origin in finite-time.

- Such stability property can be verified through any continuous positive definite $\mathbf{r}$-homogeneous function $V$ of any degree $m \in \mathbb{R}_{>0}$ (with weights $\mathbf{r}$ ).

- D-homogeneity of degree $\nu \in(0,1)$ itself prevents global asymptotic stability of the origin when the restriction $f(x)=0 \Leftrightarrow x=0$ holds. Note that in this case there are no solutions converging to the origin.

\section{D-homogeneous systems of degree $\nu=1$}

We have seen that for D-homogeneity degrees $n>1$ and $\nu \in(0,1)$ there cannot be global asymptotic stability of the system's origin if $f(x)=0 \Leftrightarrow x=0$ holds. However the proven stability properties only depend on the D-homogeneity degree of the system and do not use some other features as the system parameters, for example. On the other hand, for the case $\nu=1$, global asymptotic stability is possible, for example, observe that if (1) is linear, then its D-homogeneity degree is $\nu=1$ and the asymptotic stability property relies on the values of the system parameters. It is important to mention that when (1) is $D_{\mathbf{r}}$-homogeneous of degree $\nu=1$ and the origin is an asymptotically stable equilibrium point, the existence of a $\mathbf{r}$-homogeneous Lyapunov function is guaranteed [14].
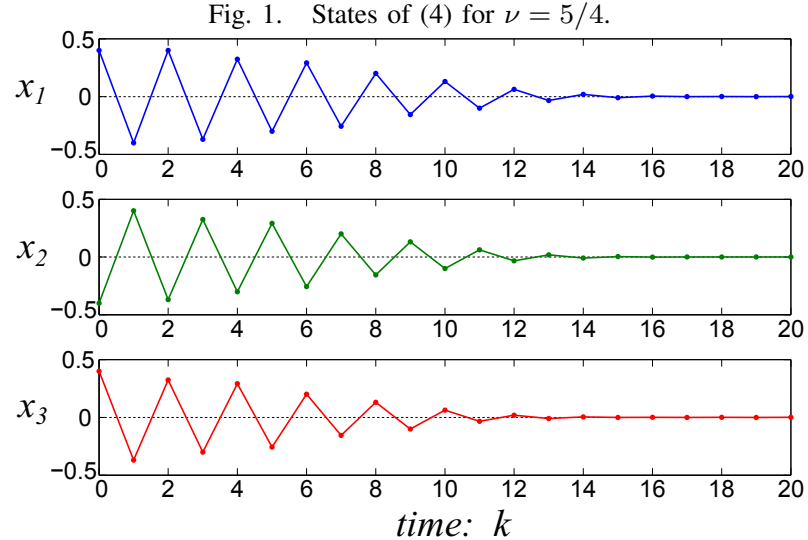

\section{EXAMPLES}

Consider the discrete-time autonomous system

$$
\begin{aligned}
x_{1}(k+1) & =x_{2}(k) \\
x_{2}(k+1) & =x_{3}(k) \\
& \vdots \\
x_{n-1}(k+1) & =x_{n}(k) \\
x_{n}(k+1) & =u
\end{aligned},
$$

and the control input

$$
u=-\sum_{i=1}^{n} a_{i}\left\lceil x_{i}(k)\right\rfloor^{\rho_{i}}, \quad \rho_{i}=\nu^{n-i+1}, \quad a_{i} \in \mathbb{R}_{>0},
$$

the closed loop (2), (3) is a $D_{\mathbf{r}}$-homogeneous system of degree $\nu$ with $\mathbf{r}=\left[r_{1}, r_{1} \nu, \ldots, r_{1} \nu^{n-1}\right]^{\top}$ for any $r_{1} \in \mathbb{R}_{>0}$. According to Theorem 2 and Theorem 3, the origin of (2), (3), is locally asymptotically stable for $\nu>1$ and practically stable for $\nu \in(0,1)$. Then, the selection of the parameters $a_{i} \in \mathbb{R}_{>0}$ cannot influence the qualitative behaviour of the system. However, when $\nu=1$, the system is linear and the parameters $a_{i}$ must be correctly designed to guarantee asymptotic stability of the origin. In the particular case of $n=3$, the closed loop (2), (3) is (we are omitting the dependence of $k$ on the right side of the equation)

$$
\begin{aligned}
& x_{1}(k+1)=x_{2} \\
& x_{2}(k+1)=x_{3} \\
& x_{3}(k+1)=-a_{1}\left\lceil x_{1}\right\rfloor^{\nu^{3}}-a_{2}\left\lceil x_{2}\right\rfloor^{\nu^{2}}-a_{3}\left\lceil x_{3}\right\rfloor^{\nu}
\end{aligned}
$$

We consider $a_{1}=a_{2}=a_{3}=3 / 2$ in (4). Observe that in the linear case $(\nu=1)$ these gains would render the origin unstable.

Figure 1 shows the local asymptotic stability of the origin by choosing $\nu=5 / 4$ and setting the initial conditions $x_{1}(0)=2 / 5, x_{2}(0)=-2 / 5, x_{3}(0)=2 / 5$ (these initial conditions were chosen arbitrarily but close to the origin).

Figures 2 and 3 show the practical stability of (4) for $\nu=$ $3 / 4$ with the initial conditions $x_{1}(0)=80, x_{2}(0)=-80$, $x_{3}(0)=80$ (these initial conditions were chosen arbitrarily but far from the origin). In Figure 4, local instability of the origin (as stated in Theorem 3) is verified for $\nu=3 / 4$. 

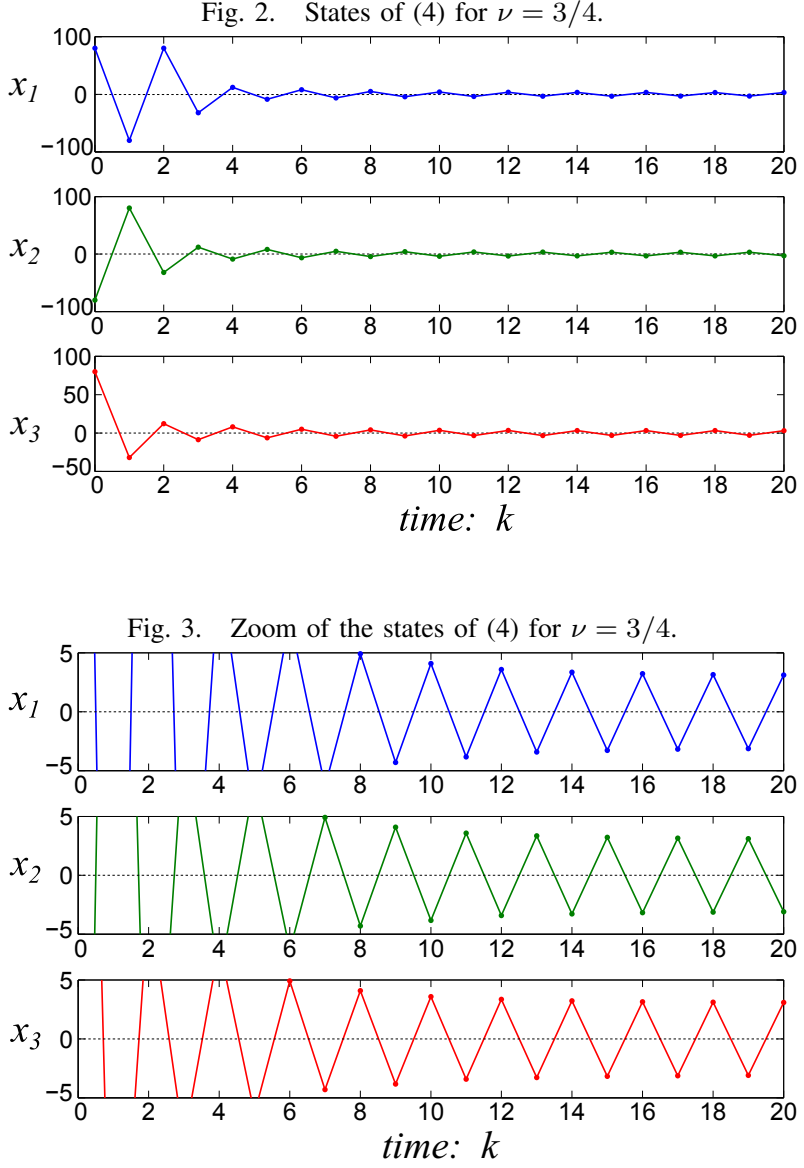

Now we sketch a possible application of D-homogeneity for global asymptotic stabilization by means of the controller (3) and the following switching control strategy:

$$
\text { Set } \nu=\left\{\begin{array}{ll}
3 / 4 & \text { if } \quad|x(k)| \geq \varepsilon \\
5 / 4 & \text { if } \quad|x(k)|<\varepsilon
\end{array}\right. \text {. }
$$

For this example we choose $\varepsilon=1 / 2$ based on simulations, however, an analytic selection of $\varepsilon$ should be done using the estimations of the attraction domains and the practical stability region from Theorems 2 and 3. In order to compare this strategy with the linear case $\nu=1$, we select $a_{1}=$ $a_{2}=a_{3}=1 / 2$ for both control schemes. Figures 5 and 6 show the responses of the linear and the switched schemes, respectively. Finally, Figure 7 shows the behaviour of $\ln (|x(k)|)$, this is to compare the convergence rate of both schemes. Note that the switched one is faster than the linear one.

\section{CONCLUSIONS}

In this work D-homogeneity is propposed. This is a notion of homogeneity for nonlinear discrete-time systems, which generalizes the previously introduced frameworks of [7] and [14]. D-homogeneity allows the trajectories of Dhomogeneous discrete-time systems to be scaled, as in the continuous-time case. Contrarily the latter, in the discretetime this does not lead to a global stability/instability of the
Fig. 4. Instability of the origin of (4) for $\nu=3 / 4$.
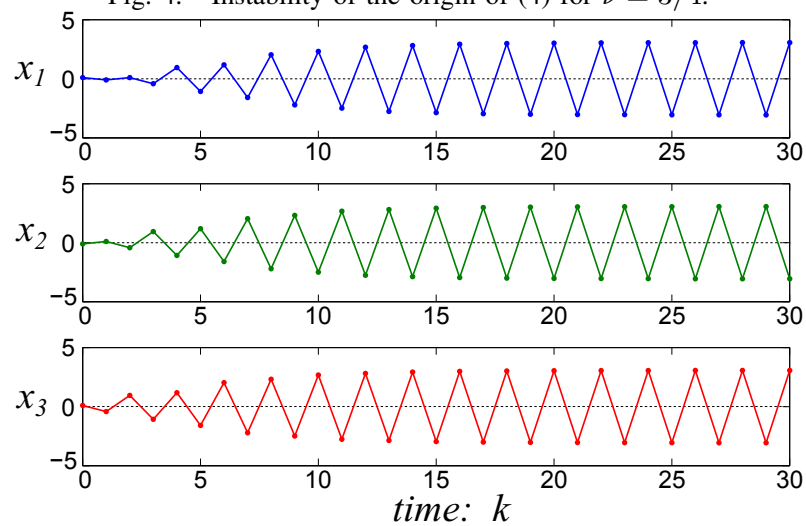

Fig. 5. States of (4) for $\nu=1$.
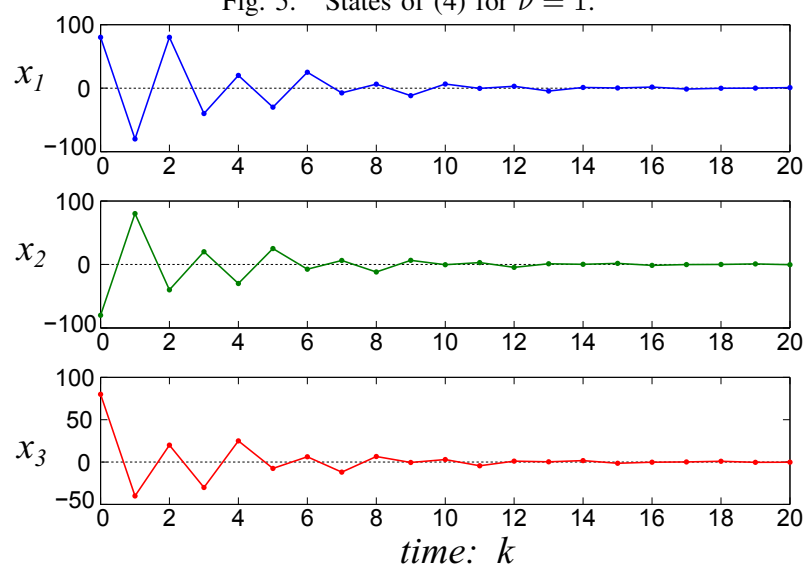

system, but it simplifies greatly the local stability analysis of the system, since it becomes a function of D-homogeneity degree only. In addition, it is shown that there is no problem with selection of Lyapunov functions for D-homogeneous discrete-time systems (its adjustment allows the domain of attraction or repulsion to be better evaluated). The theoretical findings are illustrated by a nonlinear stabilization control design with the results of simulations confirming efficiency of the concept.

The directions of future research include: extension of the definitions of D-homogeneity to generalized dilation operators; analysis of robustness properties of D-homogeneous systems; investigation of general schemes for control by switching the D-homogeneity degrees; introduction of local approximations and limit homogeneity; and studying the possibility to design D-homogeneous observers (predictors).

\section{ACKNOWLEDGMENTS}

This work is supported in part by: HoTSMoCE Inria associate team program, ANR Finite4SoS (ANR 15 CE23 0007); by the Government of Russian Federation (Grant 074U01); by the Ministry of Education and Science of Russian Federation (Project 14.Z50.31.0031); by the financial support from PAPIIT-UNAM (Programa de Apoyo a Proyectos de Investigación e Innovación Tecnológica), project IN113617; 

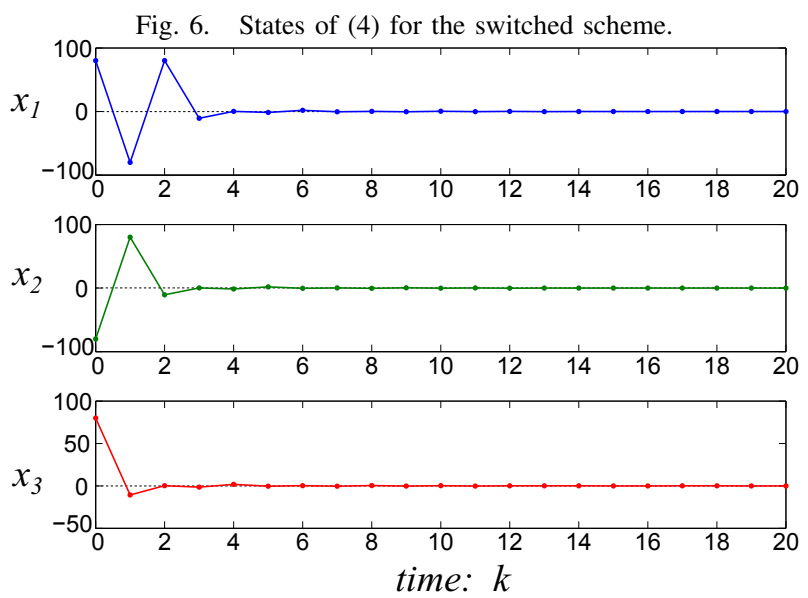

Fig. 7. Comparison of convergence rates of Linear and Switched schemes.

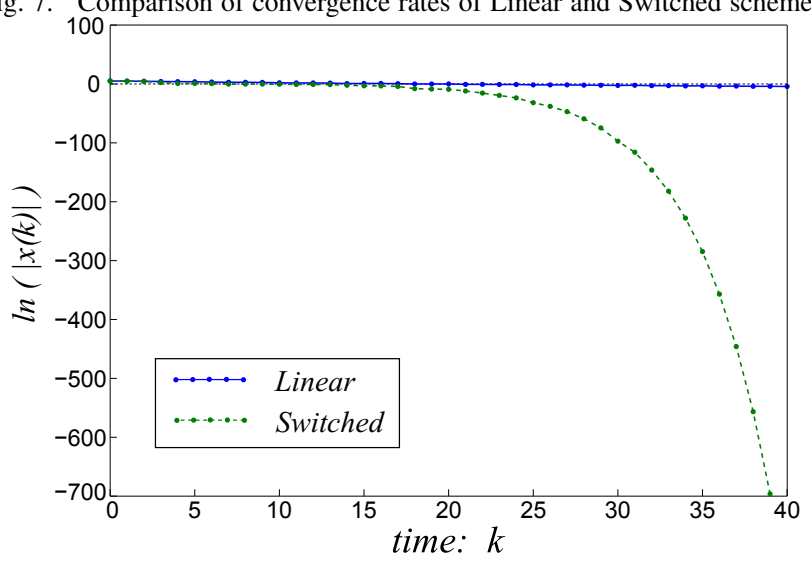

Fondo de Colaboración II-FI UNAM, Project IISGBAS100-2015; CONACyT (Consejo Nacional de Ciencia y Tecnología), project 241171 and CVU 371652.

\section{REFERENCES}

[1] R. P. Agarwal. Difference Equations and Inequalities: Theory, Methods, and Applications. Pure and Applied Mathematics. Marcel Dekker, New York, 2nd. edition, 2000.

[2] A. Bacciotti and L. Rosier. Liapunov functions and stability in control theory. Communications and Control Engineering. Springer, Berlin, 2nd edition, 2005.

[3] E. Bernuau, D. Efimov, W. Perruquetti, and A. Polyakov. On homogeneity and its application in sliding mode control. Journal of the Franklin Institute, 351(4):1866-1901, 2014.

[4] P. S. Bhat and S. D. Bernstein. Geometric homogeneity with applications to finite-time stability. Mathematics of Control, Signals and Systems, 17(2):101-127, 2005.

[5] S. Bhat and D. S. Bernstein. Finite-time stability of homogeneous systems. In American Control Conference, 1997. Proceedings of the 1997, volume 4, pages 2513-2514 vol.4, Jun 1997.

[6] W. Hahn. Stability of Motion. Springer Berlin Heidelberg, 1967.
[7] H. Hammouri and S. Benamor. Global stabilization of discrete-time homogeneous systems. Systems \& Control Letters, 38(1):5-11, 1999.

[8] H. Hermes. Homogeneus coordinates and continuous asymptotically stabilizing feedback controls, in, Differential Equations, Stability and Control (S. Elaydi, ed.), volume 127 of Lecture Notes in Pure and Applied Math., pages 249-260. Marcel Dekker, Inc., NY, 1991.

[9] Y. Hong. $H_{\infty}$ control, stabilization, and input-output stability of nonlinear systems with homogeneous properties. Automatica, 37(6):819829, 2001.

[10] A. Levant. Homogeneity approach to high-order sliding mode design. Automatica, 41(5):823-830, 2005.

[11] Y. Orlov. Finite time stability of homogeneous switched systems. In Decision and Control, 2003. Proceedings. 42nd IEEE Conference on, volume 4, pages 4271-4276, Dec 2003.

[12] L. Rosier. Homogeneous Lyapunov function for homogeneous continuous vector field. Systems \& Control Letters, 19(6):467-473, 1992

[13] R. Sepulchre and D. Aeyels. Homogeneous Lyapunov functions and necessary conditions for stabilization. Mathematics of Control, Signals and Systems, 9(1):34-58, 1996.

[14] S. E. Tuna and A. R. Teel. Discrete-time homogeneous Lyapunov functions for homogeneous difference inclusions. In 2004 43rd IEEE Conference on Decision and Control (CDC) (IEEE Cat. No.04CH37601), volume 2, pages 1606-1610 Vol.2, Dec 2004.

[15] V. I. Zubov. Methods of A. M. Lyapunov and their applications. Groningen: P. Noordho: Limited, 1964. 